\title{
EVALUATION OF CRESTAL BONE CHANGES AROUND DELAYED IMPLANT IN RESPONSE TO DIODE LASER THEARAPY: A RANDOMIZED CONTROLLED CLINICAL TRIAL.
}

\author{
Saleh Ahmed Saleh Ahmed Bakry*, Heba M. Kamel ${ }^{* *}$ and Sherief Awad ${ }^{* * *}$
}

\begin{abstract}
Purpose: Dental implants have become an essential part in the field of dentistry. One of the commonly used criteria for evaluation of dental implant success is the marginal bone changes around the dental implant. Its maintenance plays an important role in the survival of dental implants. One of the major causes of marginal bone loss is the long-term peri-implant tissue inflammation, which is attributed to poor maintenance of the oral hygiene measures.
\end{abstract}

Aim: The aim of the study was to evaluate crestal bone changes around delayed dental implants subjected to low intensity laser application (LLLT) compared to dental implants without laser application.

Patients and methods: This was a randomized controlled clinical trial conducted on twelve patients with twelve missing premolar maxillary teeth which needed to be replaced with dental implants. The Patients were randomly divided into 2 groups. Group A (non-laser group) received endosseous root form implant, and the healing phase was left to progress spontaneously without any intervention while Group B (Laser group) received endosseous root form implant followed by low intensity laser application (LLLT). Each patient received a total number of fifteen laser sessions, one was performed two weeks before implant placement and the other sessions were performed every two weeks during the six months follow up period, started 14 days after implant placement. Each laser session was performed as two sessions every day on two alternative days. In each session, the laser device was adjusted to deliver a diode laser beam with an output power of 2-watt, wavelength of 980 and a frequency of $300 \mathrm{hz}$ for 5 minutes.

Follow up was performed at base line (Day of implant placement), 2 weeks, 3 and 6 months postoperatively after implant placement. Clinical parameters assessed were probing depth (PD) and the distance between implant shoulder and mucosa (DIM). Radiographic parameter measured using paralleling periapical $\mathrm{x}$-ray was the distance between implant shoulder and bone (DIB), which represented crestal bone level.

After six months, the patients were recalled again and the healing cap was removed to place the abutment of the implant and a prosthetic part was fabricated.

\footnotetext{
* Assistant professor of Oral and Maxillofacial Surgery, Faculty of Dentistry, Cairo University.

** Lecturer of Oral and Maxillofacial Surgery, Faculty of Dentistry, Cairo University.

*** Assistant Researcher, Surgery and Oral Medicine Dep., Oral and Dental Research Division, National Research Centre.
} 
Results: For the clinical parameters measured, including the PD and DIM, it was shown that the LLLT had no effect on the results as no significant difference in pocket depth was found between the two groups. For the DIB which was measured radiographically on paralleling periapical radiograph, the mean crestal bone loss was $0.4 \mathrm{~mm}$ after 6 months of implant placement in the Laser group versus $0.7 \mathrm{~mm}$ after 6 months in the non-laser group. This result showed no statistically significant difference between the two groups.

Conclusion: The results of our study concluded that the LLLT has no effect on the implant success rate, as it did not affect neither soft nor hard tissue around dental implants. However, further studies are needed in this subject, with larger sample size and longer follow-up periods.

KEYWORDS: Delayed implants, Laser, LLLT, marginal bone loss.

\section{INTRODUCTION}

Dental implants have become an essential part in the field of dentistry. It helped to achieve many oral therapies that have been impossible with the conventional methods. (1) One of the commonly used criteria for evaluation of dental implant success is the marginal bone changes around the dental implant. (2) Its maintenance plays an important role in the survival of dental implants. ${ }^{(3,4)}$ Also, peri-implant crestal bone has a major role in the overlying soft tissue integrity. ${ }^{(5)}$ One of the major causes of marginal bone loss is the long-term periimplant tissue inflammation, which is attributed to poor maintenance of the oral hygiene measures. ${ }^{(6,7)}$

Oh and his coworkers 2002 developed a hypothesis (the biologic width hypothesis) to explain the early bone loss around dental implants. They claimed that the bone resorption around dental implants happens in a way that is similar to the biologic width that surrounds the natural tooth. They also explained that some secondary factors could cause early bone loss around dental implants, such as: the mechanical and surgical trauma that occurs during insertion of the dental implant, occlusal factors, implant collar design, in addition to the microgaps and micromovements. ${ }^{(8)}$

Implants are defined to be successful when the vertical bone loss around the implant is less than 1 $\mathrm{mm}$ in the first year, and $0.2 \mathrm{~mm}$ in the following years. ${ }^{(9)}$
For the implants in which two-piece method is used, the early peri-implant bone resorption usually starts after doing the second surgery. As, in this surgery, the implant becomes exposed to the oral cavity.

It was reported in the literature that marginal bone loss occurs usually during the first year of function, and the biologic width which consists of epithelial attachment and connective tissue forms around the implant creating a mucosal barrier against the oral flora. This explains why resorptions do not continue after the first year of function. ${ }^{(11,12)}$

Nowadays, Laser technology has gained more popularity in all fields of dentistry. With the expanding field of dental implants, surgical lasers are now widely used in a variety of ways, ranging from implant insertion, secondary surgery to expose the implant, gingival management and the treatment of peri-implantitis. ${ }^{(13-16)}$

The term LASER is an abbreviation for Light Amplification by Stimulated Emission of Radiation. In the field of dental medicine, lasers are used in two main ways, biostimulation and surgery. Biostimulation is the activation of regenerative and healing processes. Lasers used for this purpose are called low-level laser therapy (LLLT), and they operate under $500 \mathrm{Mw}$. On the other hand, lasers that work beyond the $500 \mathrm{Mw}$ are used as high intensity laser therapies (HILT), which are also known as 
surgical lasers due to their ability to cut tissues. For the purpose of biostimulation, the diode and helium neon $(\mathrm{HeNe})$ lasers are the most commonly used types depending on their active medium. ${ }^{(17-20)}$

Diode is considered a solid active medium laser. It is manufactured from a semiconguctor crystal using a combination of aluminum or indium, gallium, and arsenic.

The wavelength of diode laser used in the field of dentistry ranges from $800 \mathrm{~nm}$ for an aluminum medium, $980 \mathrm{~nm}$ for an indium medium, to a near infrared portion of the invisible non ionizing spectrum. ${ }^{(19)}$

The surgical unit of the diode laser is compact, small and portable. Most of the diode lasers used in dentistry have a flexible optic fiber in order to focus the treatment beam to the target area. This optic fiber usually comes in the form of an easily handled headpiece. ${ }^{(13,14)}$

Diode laser also has the advantages of low cost and the safety use with the dental implant as it does not damage its titanium surface. ${ }^{(21)}$ LLLT is a noninvasive treatment modality that uses lowlevel (lowpower) lasers or light emitting diodes. (22-24) LLLT is known to enhance bone healing. ${ }^{(24)}$ In dentistry, LLLT has been used for different modality as biostimulation of wounds, collagen synthesis, and fibroblast proliferation. ${ }^{(24-26)}$

The aim of our study is to evaluate crestal bone changes around delayed dental implants subjected to LLLT compared to dental implants without laser application.

\section{PATIENTS AND METHODS}

\section{Study Design}

This was a randomized controlled clinical trial conducted on twelve patients with twelve missing premolar maxillary teeth which needed to be replaced with dental implants. The Patients were randomly divided into 2 groups. Group A (non-laser group) received endosseous root form implant. The healing phase was left to progress spontaneously without any intervention and Group B (laser group) received endosseous root form implant followed by low intensity laser application (LLLT). The study was conducted in the Faculty of Dentistry, Cairo University, and a detailed informed written consent including the details of surgery and the possible complications was obtained from all patients.

\section{Eligibility Criteria}

The selected patients had missing premolar maxillary teeth which needed to be replaced with dental implants. All patients had sufficient bone width $(6 \mathrm{~mm}$ buccolingual width, and $8 \mathrm{~mm}$ mesiodistally) to allow for placement of implants with implant diameter of $3.7 \mathrm{~mm}$ and implant length of $12 \mathrm{~mm}$.

\section{The patients included in the study fulfilled the fol- lowing criteria:}

- All patients were willing and accepting to go for minor surgical procedure.

- Age ranging from 30-50 years.

- Non- smokers.

- Non medically compromised patients.

- The patients were not under any systemic medication.

\section{Randomization}

The patients were allocated randomly in the two groups using allocation ratio 1:1. The randomization was performed using 12 opaque envelopes, 6 for group A and 6 for group B.

\section{Preoperative Preparation}

Medical and dental history were documented. Clinical assessment was performed through observing the patient's occlusion, inter arch space, 
oral hygiene and available bone. Digital examination of the covering mucosa was performed by applying finger pressure, to detect any gingival swelling, tender areas or extremely thin mucosa. Extraoral examination included inspection and palpation to detect any tenderness, lymph node enlargement, swelling or asymmetry.

As regarding radiographic examination, preoperative Digital panoramic radiographs were taken to determine the available bone height, and to exclude the presence of any remaining roots or residual infections (Figure 1).

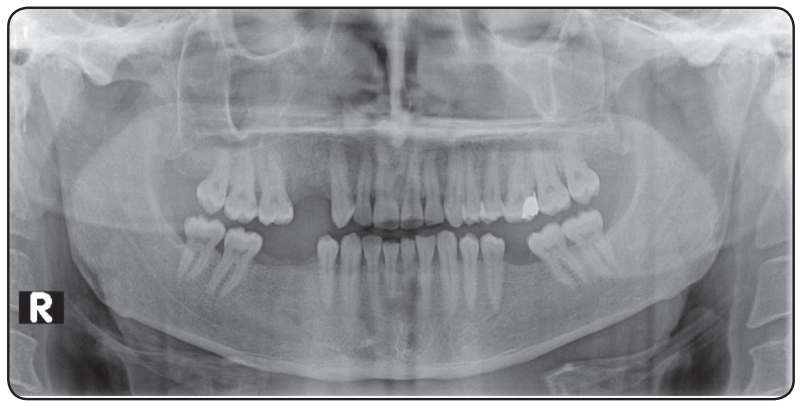

Fig. (1) Preoperative digital panoramic radiograph

\section{Patient grouping:}

\section{Group A (non-laser group):}

This group received endosseous root form implant (SwissPlus implant system, Zimmer Dental Inc, Carlsbad CA, USA) The healing phase was left to progress spontaneously without any intervention.

\section{Group B (laser group):}

This group received endosseous root form implant (SwissPlus implant system, Zimmer Dental Inc, Carlsbad CA, USA) followed by low intensity laser application (LLLT). The device used was Smart M lasotronix Pro diode laser. Smart M Pro is used for cutting, coagulation and tissue vaporization. High-end optical system and the availability of different light sources as well as flexible fiber optic with a variety of handpieces provides a unique application scope in microsurgery and biostimulation. The Smart M Pro available in many versions, differing in power and wavelength, it can be used in a small practice as well as in a large clinic. Due to its inherent properties and current technological advancement, the most versatile and efficient wavelength of a semiconductor diode module is $980 \mathrm{~nm}$. Additionally, the laser beam provides safe, "cold" biostimulation and photoactivated disinfection in relatively short time of therapy. Due to proper applicators and parameters, the healing process of any wounds can be significantly accelerated (Figure 2). Each patient received a total number of fifteen laser sessions, one was performed two weeks before implant placement and the other sessions were performed every two weeks during the six months follow up period, started 14 days after implant placement. Each laser session was performed as two sessions every day on two alternative days. In each session, the laser device was adjusted to deliver a diode laser beam with an output power of 2-watt, wavelength of 980 and a frequency of $300 \mathrm{hz}$ for 5 minutes.

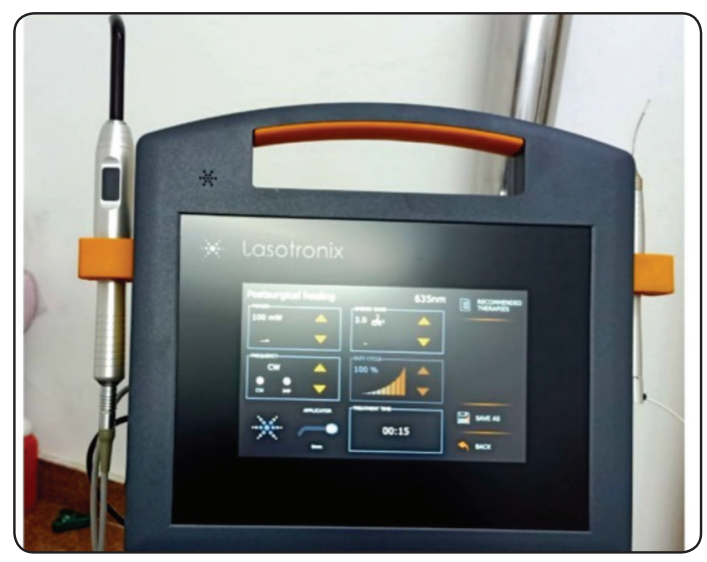

Fig. (2) SMART M Pro laser device.

\section{Surgical procedure:}

Immediately before surgery the patient was allowed to rinse with Chlorohexidine Gluconate $0.1 \%$ mouth wash (Antiseptol, Kahira Pharma 
Co, Cairo, Egypt). Buccal and palatal infiltration were given using Articane 4\% with 1: 100000 Epinephrine (Ubistesin TM forte, 3M ESPE, Germany). A standard crestal incision using no. 15 blade was done extending one tooth mesial and one tooth distal to the implant site. A full thickness mucoperiosteal flap was reflected buccally and palatally using a suitable mucoperiosteal elevator to expose the implant recipient site.

\section{Implant placement:}

A round bur was used first to gain access to the bone. Initial $2.1 \mathrm{~mm}$ drill was used to drill through the bone under copious amount of saline irrigation. Pilot drill of $2.3 \mathrm{~mm}$ diameter was used. Drilling continued till the planned depth. Drills of sequential diameters were used till reaching the planned final diameter $3.7 \mathrm{~mm}$ and length $12 \mathrm{~mm}$. The implant was screwed using ratchet wrench till intra-osseous portion of implant was completely inserted in the bone and $2 \mathrm{~mm}$ height healing cap was screwed to the implant.

After the implant was placed to the final position, the flap was closed around the healing cap by a tension free closure using 3-0 black silk suture to prevent wound dehiscence (Figure 3). Postoperatively, the patients were instructed to use extraoral ice packs for the first postoperative six hours. Non-steroidal anti-inflammatory analgesic (Diclofenac potassium 50mg, Catafast 50mg tablets, Novartis Pharma AG, Cairo, Egypt) was prescribed three times daily for three days. Antibiotic (clindamycin 300mg, Clindam $300 \mathrm{mg}$ capsules, Sigma pharmaceutical industries, Egypt) was prescribed three times daily for five days. Regular oral hygiene measures were resumed after a day from the day of surgery. Sutures were removed after 7 days. The day of implant placement will be defined as day 0 . All patients were recalled 2,12 , and 26 weeks postoperatively for clinical and radiographic evaluation.
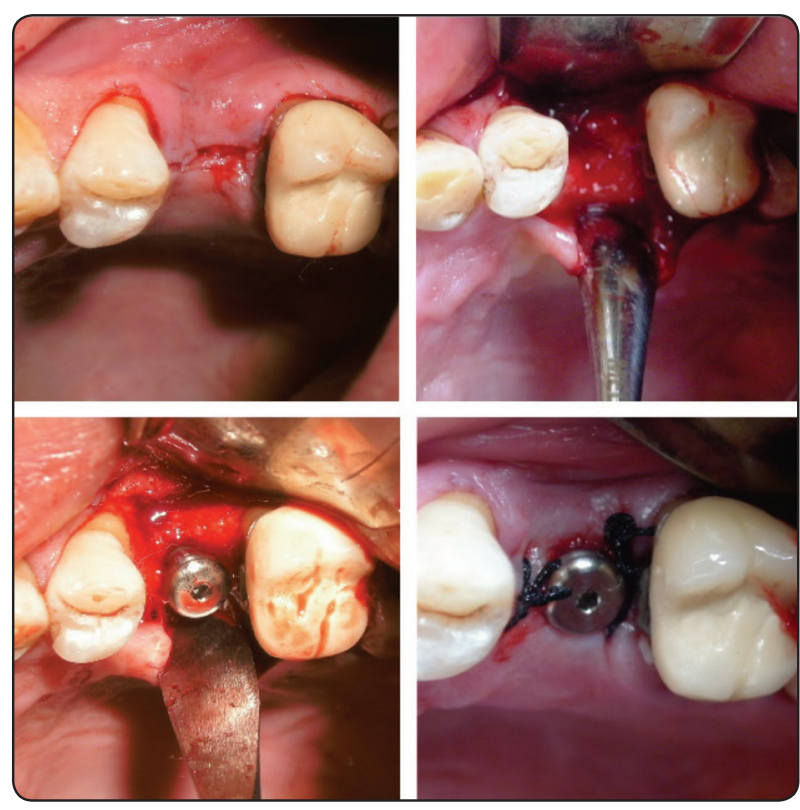

Fig. (3) Photograph showing implant placement with healing cap into the osteotomy site.

\section{Laser application:}

For Group B patients were instructed to attend the low intensity laser application sessions according to the predetermined schedule. The implant site was exposed to low intensity laser irradiation (diode laser). The total number of main sessions during healing was fifteen, performed as two sessions every day on two alternative days. In each session, the laser device was adjusted to deliver a laser beam with an output power of 2 watt and a frequency of 300 hz for 5 minutes. During the pre-adjusted time (five minutes), the buccal, the lingual as well crestal surfaces were allowed to receive the laser beam. Laser beam was continuously delivered from the tip of the laser applicator and exposing the target surface with the tip touching the tissues and directed towards the implant site. The applicator tip was moved in a continuous slow circular motion to assure full exposure of the target surface to the laser beam.

After six months, the patients were recalled again and the healing cap was removed to place the abutment of the implant and a prosthetic part was fabricated. 


\section{Postoperative assessment:}

- The day of implant placement was defined as day 0 .

- At 2 weeks, 3 and 6 months after implant placement the following clinical and radiographic parameters were assessed.

\section{A. Clinical follow up:}

1. The implant site was checked for signs of infection, pain or discomfort.

2. Probing depth (PD, in $\mathrm{mm}$ ) at four aspects around the implants was measured. For each implant, one PD value was calculated based on the average of the four obtained values (Figure 4).

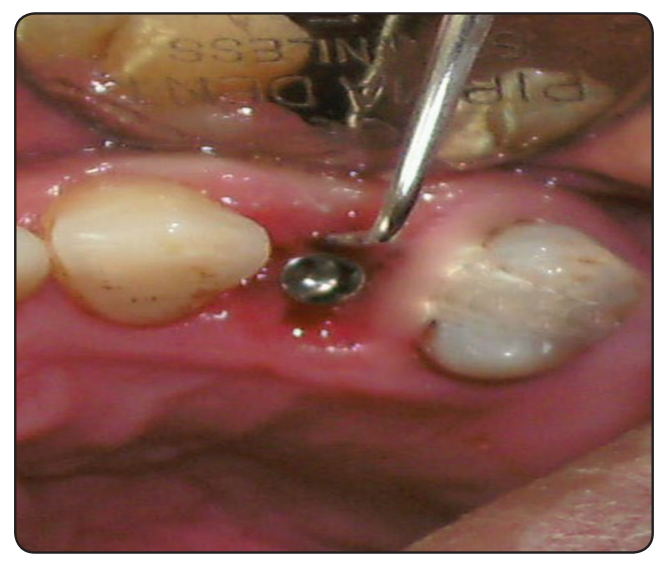

Fig. (4) Clinical Photograph showing the PD

3. The distance between the implant shoulder and the mucosal margin (DIM, in $\mathrm{mm}$ ) at four aspects around the implants. A submucosal implant shoulder was recorded with a negative DIM value. For each implant, one DIM value was calculated based on the average of the four obtained values. (Figure 5)

\section{B. Radiographic follow up:}

1. Periapical radiographs were taken using minray x-ray machine (Soredex, Finland.). The exposure parameters for the baseline and follow-up images were standardized and set as follows; $70 \mathrm{kVp}, 7 \mathrm{~mA}$ and 0.04 exposure time.

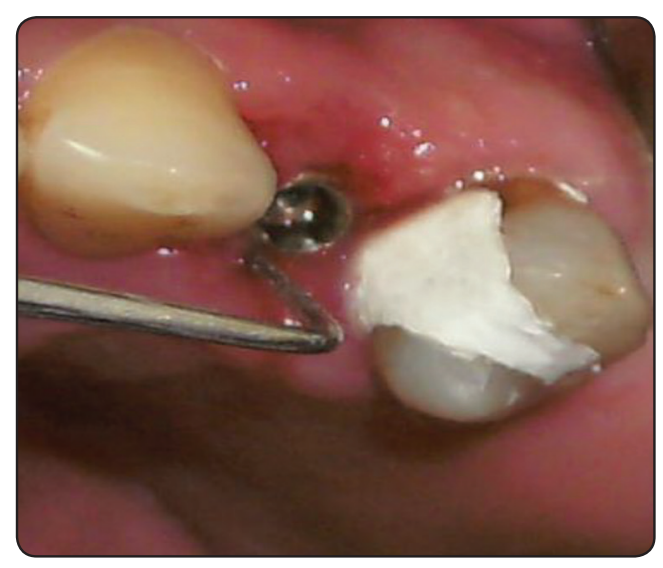

Fig. (5) Clinical Photograph showing the DIM

2. The standardized long cone paralleling technique was used to perform all images using Rinn XCP film holder (Rinn XCP film holder, Rinn Corporation, USA) which consists of interchangeable plastic bite blocks, a plastic aiming ring and a metallic indicator arm. For standardization and reproducibility of geometric parameters during radiographic exposure, for every patient, individually customized radiographic templates were constructed and used in conjunction to the holding devices (Figure 6 and 7).

3. Digitized images were manipulated using a specially designed software of the Digora system (Digora: the photo stimulus phosphor digital radiographic system, USA).

- The distance between the implant shoulder and the first visible bone to implant contact (DIB) was measured (in $\mathrm{mm}$ ) at the mesial and distal aspect of each implant using digitized periapical radiographs (Digora: the photo stimulus phosphor digital radiographic system, USA) which were taken with the long-cone technique at 0,2,12 and 26 weeks .For each implant, one DIB value was calculated based on the average of the mesial and distal values.

- All data were collected and tabulated for statistical analysis. 


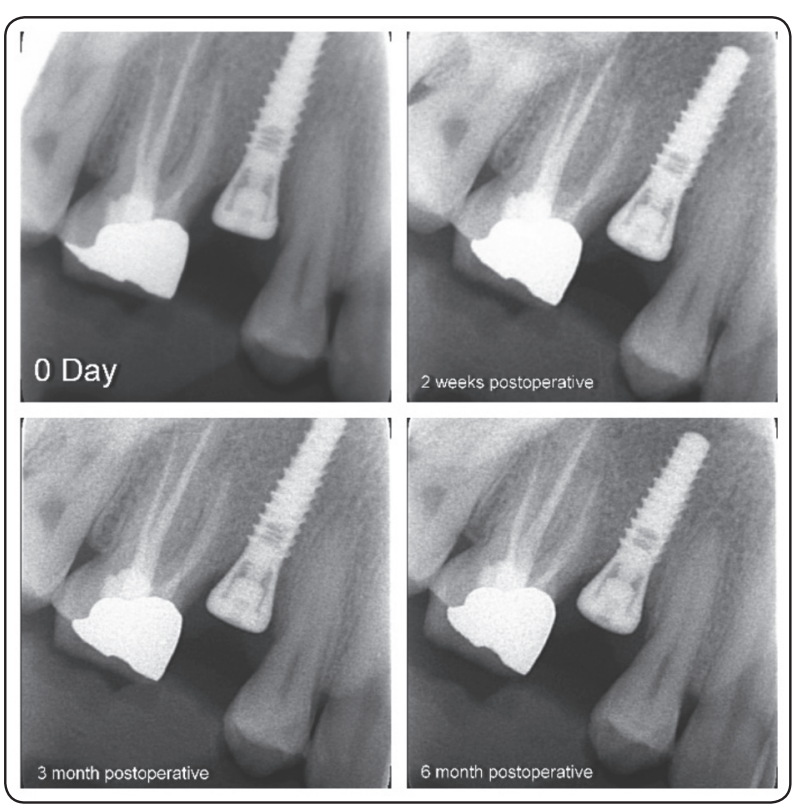

Fig. (6) Digitized radiographs of the radiographic follow up of the non-Laser group.

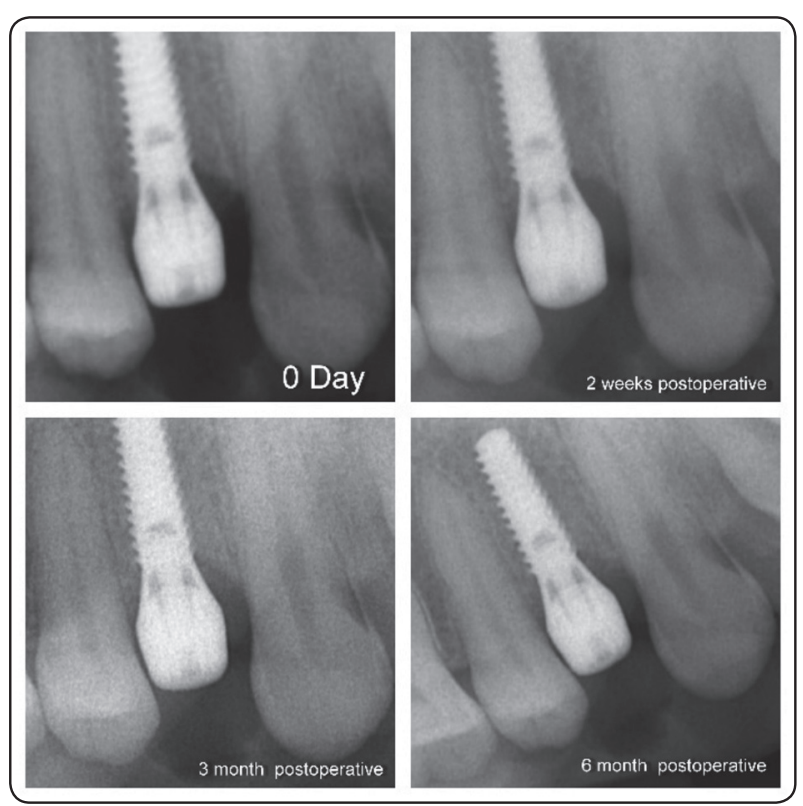

Fig. (7) Digitized radiographs of the radiographic follow up of Laser group.

\section{Statistical analysis}

Data were presented as mean and standard deviation (SD) values. Data showed non-parametric distribution so Mann-Whitney U test was used to compare between the two groups. This test is the non-parametric alternative to Student's t-test. Wilcoxon signed-rank test was used to study the changes by time within each group. This test is the nonparametric alternative to paired t-test.

The significance level was set at $\mathrm{P} \leq 0.05$. Statistical analysis was performed with IBM (IBM Corporation, NY, USA) SPSS (SPSS, Inc., an IBM Company) Statistics Version 20 for Windows.

\section{RESULTS}

The study was conducted on 12 patients with 12 missing premolar maxillary teeth who needed to replace their missing teeth with dental implants. The present study was done to evaluate the clinical and radiographic changes around non-submerged titanium implants with the objectives of evaluating the clinical parameters as probing depth (PD), the distance between implant shoulder and mucosal margin (DIM), also radiographic parameters as distance between implant shoulder and first visible bone level (DIB) for the two group.

\section{Clinical Parameters:}

\section{Probing depth (PD):}

As regarding changes by time in each group, it was found that there was non-statistically significant decrease in mean PD measurement after 2 weeks, 3 and 6 months neither in group A (non-laser group) nor in group B (Laser group).

When we compared the two groups in each time period, non-laser group showed statistically significantly higher mean PD measurement than Laser group in the base line measurement. However, after 2 weeks, 3 months and 6 months; there was no statistically significant difference between mean PD measurements in the two groups (table 1). 
TABLE (1) The mean, standard deviation (SD) values and results of Mann-Whitney $U$ test for comparison between PD measurements of the two groups.

\begin{tabular}{|l|c|c|c|c|c|}
\hline \multirow{2}{*}{ Group } & \multicolumn{2}{|c|}{ Laser } & \multicolumn{2}{c|}{ Control } & \multirow{2}{*}{$P$-value } \\
\cline { 2 - 5 } & Mean & SD & Mean & SD & \\
\hline Base line & 2.9 & 0.4 & 4.9 & 1.6 & $0.034^{*}$ \\
\hline 2 weeks & 2.8 & 0.3 & 4.3 & 2.5 & 0.266 \\
\hline 3 months & 2.3 & 0.4 & 3.3 & 1.7 & 0.354 \\
\hline 6 months & 2.8 & 0.4 & 3.2 & 1.2 & 0.857 \\
\hline
\end{tabular}

*: Significant at $P \leq 0.05$

\section{Distance between implant shoulder and mu- $\operatorname{cosa}(\mathrm{DIM})$ :}

As regarding changes by time in each group, both groups had the same results. There was nonstatistically significant increase in mean DIM measurement after 2 weeks. Also, there was nonstatistically significant decrease in mean DIM measurement after 3 months and 6 months.

When we compared the both groups in each time period, there was no statistically significant difference between mean DIM measurements in all the follow-up intervals (table 2).

TABLE (2): The mean, standard deviation (SD) values and results of Mann-Whitney $\mathrm{U}$ test for comparison between DIM measurements of the two groups.

\begin{tabular}{|c|c|c|c|c|c|}
\hline \multirow{2}{*}{ Group } & \multicolumn{2}{|c|}{ Laser } & \multicolumn{2}{c|}{ Control } & \multirow{2}{*}{ P-value } \\
\cline { 2 - 6 } & Mean & SD & Mean & SD & \\
\hline Base line & 3.1 & 0.5 & 3.2 & 0.4 & 0.586 \\
\hline 2 weeks & 3.6 & 0.5 & 3.3 & 0.6 & 0.476 \\
\hline 3 months & 2.9 & 0.4 & 2.3 & 0.7 & 0.266 \\
\hline 6 months & 2.4 & 0.3 & 2.3 & 1.1 & 0.589 \\
\hline
\end{tabular}

*: Significant at $P \leq 0.05$

\section{Radiographic Parameter:}

\section{Distance between implant shoulder and Bone (DIB):}

As regarding changes by time in each group, it was found that there was non-statistically significant increase in mean DIB measurement after 2 weeks, 3 and 6 months neither in group A (non-laser group) nor in group B (Laser group).

When we compared the both groups in each time period, there was no statistically significant difference between mean DIM measurements in all the follow-up intervals (Table 3 ).

TABLE (3): The mean, standard deviation (SD) values and results of Mann-Whitney $\mathrm{U}$ test for comparison between DIB measurements of the two groups.

\begin{tabular}{|c|c|c|c|c|c|}
\hline \multirow{2}{*}{ Group } & \multicolumn{2}{|c|}{ Laser } & \multicolumn{2}{c|}{ Control } & \multirow{2}{*}{ P-value } \\
\cline { 2 - 5 } & Mean & SD & Mean & SD & \\
\hline Base line & 2.4 & 0.6 & 2.4 & 1.3 & 0.724 \\
\hline 2 weeks & 3 & 0.9 & 3.3 & 1.5 & 0.724 \\
\hline 3 months & 3 & 1.1 & 3.1 & 1.7 & 1.000 \\
\hline 6 months & 2.8 & 1 & 3.1 & 1.3 & 0.724 \\
\hline
\end{tabular}

*: Significant at $P \leq 0.05$

\section{DISCUSION}

Osseointegration plays the main role in dental implants success and it is defined as the microscopic evidence of direct contact between bone and implant surface. Osseointegration is basically a wound healing process, thus all factors that would interfere with normal healing, such as infection and overload, may contribute to implant failure. ${ }^{(27,28)}$

The survival rate of dental implants depends mainly on the intimate integration between the implant surface and the surrounding oral tissues, including both soft and hard tissues. ${ }^{(29)}$ 
In the current study, we attempted to standardize some of the factors that may affect the implant success rate such as the surgical technique and implant material, length and diameter to evaluate the effect of low intensity laser application (LLLT) on the survival rate of the dental implants. For this reason, all patients received the same treatment protocol by the same surgical team.

LLLT is knownby itsanti-inflammatory properties through different mechanisms such as: stimulation of ascorbic acid uptake by cells, improvement of mitochondrial adenosine triphosphate production and activation of lymphocytes and mast cells. LLLT also enhances local cell circulation, cell proliferation, and collagen synthesis. ${ }^{(29)}$

LLLT also stimulates the differentiation of nondifferentiated mesenchymal cells to osteoblasts that would more rapidly change to osteocytes and act as an inducer factor that improves vascularization, anti- inflammatory effect and enhanced collagen synthesis, thus enhancing the bone healing process. ${ }^{\left({ }^{30}\right)}$ This was supported by the results of Lopes CB ${ }^{(31)}$ who concluded that LLLT improves bone healing around the dental implants.

In our study, we tried to evaluate the effect of LLLT on the factors that directly affect the implant survival rate, including clinical parameters (probing depth PD, and the distance between implant shoulder and mucosa DIM); as well as the radiographic parameters which included the evaluation of marginal bone level by measuring the distance between implant shoulder and the first visible bone contact (DIB).

For the clinical parameters measured, including the PD and DIM, it was shown that the LLLT had no effect on the results. This could be attributed to that those clinical parameters are mainly related to the ability of the patient to control plaque accumulation which is directly related to peri-implant mucositis and peri-implantitis. On searching the literature, we didn't find any papers studying the effect of laser therapy on such parameters.
As regarding the radiographic parameters assessed in the present study which included evaluation of marginal bone level which by the distance between implant shoulder and the first visible bone contact (DIB), the mean crestal bone loss was $0.4 \mathrm{~mm}$ after 6 months in the Laser group versus $0.7 \mathrm{~mm}$ after 6 months in the non-laser group. This result showed no statistically significant difference between the two groups.

The results of our study were in accordance with Mandić et al ${ }^{(32)}$ who tried to observe the effect of laser treatment on implants inserted in the posterior maxilla. They thought that laser might improve the implant success rate in this particular area. Their results showed that laser might result in increasing bone formation, however still there was no significance detected.

But, our results were not in acceptance with ElKholey and EIShenaway ${ }^{(33)}$ who studied the effect of diode laser on eight patients indicated for implant placement. They evaluated the marginal bone level radiographically at 3 and 6 months. They found that there was a statistically significant difference in the marginal bone level in favor to the Laser group. This could be attributed to the different protocol of laser application used in this study.

A recent systematic review, which was published by Ali Fahd et al $2018{ }^{(34)}$ tried to assess the laser biostimulation effects on the dental implants as regarding the stability of the dental implant, the marginal bone loss, pain and bone healing inflammatory response. They concluded that the clinical trials published are very few in number, had a small sample size, the follow-up periods were short, in addition to the non-homogenous methodology and outcomes. So, they couldn't reach a solid conclusion, and they strongly recommended the publishing of more randomized clinical trials on this subject. 


\section{CONCLUSION}

The results of our study concluded that the LLLT has no effect on the implant survival rate, as it did not affect neither soft nor hard tissue around dental implants. However, further studies are needed in this subject, with larger sample size and longer follow-up periods.

\section{REFERENCES}

1. Abdul-Aziz Al-Sawai \& Hussein Labib: Success of immediate loading implants compared to conventionally-loaded implants: a literature review. Journal of Investigative and Clinical Dentistry, 7: 217-224, 2016.

2. Yun-Chi Wang, Joseph Y. K. Kan, Kitichai Rungcharassaeng, Phillip Roe, Jaime L. Lozada: Marginal bone response of implants with platform switching and non-platform switching abutments in posterior healed sites: a 1-year prospective study. Clin. Oral Impl. Res, 26, 220-227, 2015.

3. Etoz, O.A., Demetoglu, U. \& Ocak, H.: New method to increase inter-alveolar height with preservation of crestal cortical bone for implant treatment. The Journal of Oral Implantology, 40: 601-602, 2014.

4. Al Amri MD, Al-Johany SS, Al Baker AM, Al Rifaiy MQ, Abduljabbar TS, Al-Kheraif AA.: Soft tissue changes and crestal bone loss around platform-switched implants placed at crestal and subcrestal levels: 36-month results from a prospective split-mouth clinical trial. Clin. Oral Impl. Res, 28: 1342-1347, 2017.

5. Aimetti, M., Ferrarotti, F., Mariani, G.M., Ghelardoni, C. \& Romano, F.: Soft tissue and crestal bone changes around implants with platform-switched abutments placed non-submerged at subcrestal position: a 2-year clinical and radiographic evaluation. The International Journal of Oral \& Maxillofacial Implants 30: 1369-1377, 2015.

6. Kozlovsky, A., Tal, H., Laufer, B.Z., Leshem, R., Rohrer, M.D., Weinreb, M. \& Artzi, Z.: Impact of implant overloading on the peri-implant bone in inflamed and noninflamed peri-implant mucosa. Clinical Oral Implants Research 18: 601-610, 2007.

7. Elemek, E. \& Almas, K. Peri-implantitis: Etiology, diagnosis and treatment: an update. The New York State Dental Journal 80: 26-32, 2014.

8. Oh TJ, Yoon J, Misch CE, Wang HL. The causes of early implant bone loss: myth or science: J Periodontal, 73:322-333, 2002.
9. Albrektsson T, Zarb G, Worthington P, Eriksson AR. The long-term efficacy of currently used dental implants: A review and proposed criteria of success. Int J Oral \& Maxillofac Implants, 1:11-25, 1986.

10. Esma Kütan, Nilufer Bolukbasi, Esra Yildirim-Ondur, Tayfun Ozdemir: Clinical and Radiographic Evaluation of Marginal Bone Changes around Platform-Switching Implants Placed in Crestal or Subcrestal Positions: A Randomized Controlled Clinical Trial. Clinical Implant Dentistry and Related Research, 17: 2, e364,2015

11. Abrahamsson I, Berglundh T, Wennström J, Lindhe J.: The peri-implant hard and soft tissue characteristics at different implant systems. A comparative study in dogs. Clin Oral Implants Res, 7:212-220d, 1996.

12. Berglundh T, Lindhe J.: Dimension of the peri-implant mucosa. Biological width revisited. J Clin Periodontol 23:971-973, 1996.

13. Pirnat S.: Versatility of an $810 \mathrm{~nm}$ diode laser in dentistry: an overview. J Laser Health Acad, 4:1-9, 2007.

14. Lomke MA.: Clinical applications of dental lasers. Gen Dent 57:47-59, 2009

15. Yeh S, Jain K, Andreana S.: Using a diode laser to uncover dental implants in second stage surgery. Gen Dent 53:414-7, 2005 .

16. Parker S.: Surgical laser in implantology and endodontics. Br Dent J, 202:377-86, 2007.

17. Martin E. Lasers in dental implantology.: Dent Clin North Am 48:999-1015, 2004.

18. Miller RJ. Lasers in oral implantology.: Dent Pract (Cincinnati) 112-4, 2006.

19. Coluzzi DJ: Fundamentals of dental lasers: science and instruments. The dental clinics of North America, 48: 751794,2004

20. Sant'Anna EF, Araújo MTS, Nojima LI, Cunha AC, Silveira A. BL, Marquezan M.: High-intensity laser application in Orthodontics. Dental Press J Orthod. 22(6):99-109, 2017.

21. F. G. Rios \& E. R. Viana \& G. M. Ribeiro \& J. C. González \& A. Abelenda \& D. C. Peruzzo: Temperature evaluation of dental implant surface irradiated with high-power diode laser. Lasers Med Sci 31:1309-1316, 2016.

22. Avci P, Gupta A, Sadasivam M, Vecchio D, Pam Z, Pam $\mathrm{N}$, et al. Lowlevel laser (light) therapy (LLLT) in skin: Stimulating, healing, restoring. Semin Cutan Med Surg. 32:4152, 2013. 
23. Chung H, Dai T, Sharma SK, Huang YY, Carroll JD, Hamblin MR, et al.: The nuts and bolts of lowlevel laser (light) therapy. Ann Biomed Eng. 40:51633, 2012.

24. GarcíaMorales JM, TortamanoNeto P, Todescan FF, de Andrade JC Jr., Marotti J, Zezell DM, et al.: Stability of dental implants after irradiation with an 830nm lowlevel laser: A doubleblind randomized clinical study. Lasers Med Sci. 27:70311, 2012.

25. Pourzarandian A, Watanabe H, Ruwanpura SM, Aoki A, Ishikawa I.: Effect of lowlevel er:YAG laser irradiation on cultured human gingival fibroblasts. J Periodontol. 76:18793, 2005.

26. Eduardo FP, Mehnert DU, Monezi TA, Zezell DM, Schubert MM, Eduardo CP, et al.: Cultured epithelial cells response to phototherapy with low intensity laser. Lasers Surg Med. 39:36572, 2007.

27. Ratner BD: Replacing and Renewing: Synthetic materials, biomimetics and tissue engineering in implant dentistry. $\mathrm{J}$ of dental education, 65: 1340-1347, 2001.

28. Zarb GA, Albrektsson T: Osseointegration. Requirements for the periodontal ligament. Int J Periodont Rest Dent, 11:88-91, 1991 .
29. Conlan MJ, Rapley JW, Cobb CM.: Biostimulation of wound healing by lowenergy laser irradiation. A review. J Clin Periodontal, 23:4926, 1996.

30. Islam N: Effect of Low-Level Laser therapy on human bone regeneration, 2010.

31. Lopes CB, Pinheiro AL, Sathaiah S, Duarte J, Cristinamartins M.: Infrared laser light reduces loading time of dental implants: a Raman spectroscopic study. Photomed Laser Surg., 23: 27-31, 2005.

32. Mandić B., et al.: "Influence of postoperative low-level laser therapy on osseointegration of self-tapping implants into posterior maxilla. A 6-week split-mouth clinical study”. Vojnosanitetski Pregled 72.3 ,233-240, 2015.

33. El-Kholey KE and El-Shenaway H. "Role of Diode Laser in Preservation of The Marginal Bone Around Early Loaded Endosseous Implant”. Life Science Journal 9.3, 940-943, 2012.

34. Ali Fahd., et al. "Laser Biostimulation in Dental Implant Surgery: A Systematic Review of Clinical Literature". EC Dental Science. 17.6, 827-835, 2018. 